\title{
Effects of the apical suspension of the upper vagina by cervicosacropexy or vaginosacropexy on stress and mixed urinary incontinence
}

\author{
(1) WOLFRAM JÄGER, (1) ANDREAS BRAKAT, (1) SEBASTIAN LUDWIG, (1) PETER MALLMANN \\ Department of Obstetrics and Gynecology, Division of Urogynecology and Pelvic Reconstructive Surgery, University Hospital Cologne and Medical Faculty, \\ Cologne, Germany
}

\section{ABSTRACT}

Objective: Cervicosacropexy (CESA) and vaginosacropexy (VASA) are standardized surgical procedures to elevate and tighten the apical end of the vagina. A combination of CESA or VASA, and a transobturator tape was reported to cure urgency urinary incontinence (UUI). However, the efficacy of CESA or VASA in curing stress urinary incontinence (SUI) has not been investigated.

Materials and Methods: Patients with SUI were asked to specify the situation when they urinated involuntarily. All of these patients were never operated on the genital tract before. In all the patients, the uterosacral ligaments were replaced by polyvinylidenefluoride tapes of identical length. The surgical outcomes in terms of vaginal anatomy and urinary incontinence were established several weeks after surgery. SUI was subdivided into SUI 1 (urinary loss while sneezing and coughing) and SUI 2 (urinary loss while performing other activities).

Results: In addition to the anatomical effect, the apical fixation of the upper vaginal wall by CESA and VASA led to continence in 57\% of the patients. The continence (cure) rate was higher in patients with SUI 1 (73\%) than that in patients with SUI 2 (51\%). The cure rate decreased with increase in the age of the patient at surgery. Of the 161 patients, 144 (89.4\%) patients with SUI 2 were clinically having UUI. CESA and VASA led to continence in $39.1 \%$ and $29.3 \%$ of these patients, respectively.

Conclusion: CESA and VASA can establish urinary continence in $29 \%$ to $48 \%$ of patients with stress and UUI. Continence rates decreased with patients' age.

Keywords: Cervicosacropexy; mixed urinary incontinence; stress urinary incontinence; urgency urinary incontinence; vaginosacropexy

\section{INTRODUCTION}

The Integral Theory by Petros and Ulmsten states that urinary continence in women is based on the integrity of the pubourethral ligaments (PULS) and the uterosacral ligaments (USLS). ${ }^{1-4}$ This theory was supported when suburethral tapes to replace the PULs led to continence. ${ }^{5}$ In stress urinary incontinence (SUI) the increased pressure during coughing or sneezing leads to a short opening of the urethrovesical junction (UVJ), leading to loss of urine. The tapes compress the UVJ when vertical pressure

Address for Correspondence: Sebastian Ludwig, Department of Obstetrics and Gynecology, Division of Urogynecology and Pelvic Reconstructive Surgery, University Hospital Cologne and Medical Faculty, Cologne, Germany

E-mail: sebastian.ludwig@uk-koeln.de ORCID ID: orcid.org/0000-0002-9170-9546

Received: 12 November 2020 Accepted: 15 February 2021

(C) Copyright 2021 by the International Society for Pelviperineology / Pelviperineology published by Galenos Publishing House. 
is exerted on the bladder and the patient remains continent. ${ }^{1,6}$ While the replacement of the PULS by tapes is an established treatment modality for SUI the role of the USLs for continence is not defined so far.

Descensus of the uterus and the vagina is often associated with urinary incontinence. ${ }^{7,8}$ In these patients the USLs are stretched; however, it is not known if that is the cause or the effect of the descensus. The role of the USLS in urinary continence and incontinence has not been investigated in detail. ${ }^{9}$ With increasing age, the major problem of urinary incontinence is the sudden urge to void along with nearly immediate loss of urine. Urgency urinary incontinence (UUI) was primarily considered as a neurological disorder, with effects on the detrusor muscle, and was treated accordingly. ${ }^{10}$ It was observed that medical treatment could improve symptoms and reduce incontinence episodes. However, it did not lead to normal continence. ${ }^{11-13}$

Another prediction of the Integral Theory is the significance of the intact USLS. In the Petros's "bridge allegory," it was stated that both suspensions in the front and in the back must be intact to hold the road (vagina) in balance. .,3,14 $^{2}$ The USLs put tension on the upper vagina to stabilize the bladder resting on that part of the vagina. When that tension decreases, the pressure of the full bladder can stretch the vagina, especially in the upright body position.

Some women develop an instability of the UVJ with increasing age, which could lead to the sensation of "urgency" and urine Ioss. ${ }^{15,16}$ This hypothesis was supported by the finding that the elevation of this part of the vagina during clinical vaginal examination leads to the disappearance of the feeling of "urgency" in respective patients. ${ }^{1,2}$

To elevate the upper part of the vagina, we developed a surgical procedure to restore the posterior suspension of the vagina, namely the USL. ${ }^{17-20}$ Studies have reported that the length of the USL is approximately 8.5-9.3 cm..$^{21,22}$ Tapes were either sutured to the cervix through cervicosacropexy (CESA) or at the vaginal stump through vaginosacropexy (VASA) and tunneled along the uterosacral folds to the sacral bone where they were fixed. 18,23,24 In patients with stress and UUI (mixed UI) a CESA or VASA tape was placed together with a transobturator tape (TOT). ${ }^{19}$ That resulted in continence in $75 \%$ of the patients. ${ }^{25}$ It was hypothesized that this was due to the TOT effect on the stress factor and the CESA or VASA effect on the urgency factor.

In a randomized clinical trial (URGE 1), we evaluated the effectiveness of CESA or VASA in patients with UUI comparing the surgical approach with an established medical treatment. ${ }^{25}$ Several of these patients with UUI were also suffering from MUI. During the study it was observed that CESA or VASA could lead to continence also in these patients with MUI. ${ }^{25,26}$ That was interpreted as an effect of CESA or VASA on the suburethral hammock. During further treatment of patients with MUI the surgical procedures were separated. In the first step, the CESA or VASA structures were placed. If the patients were still incontinent a TOT was placed several weeks later.

In this study we identified the stress-related symptoms that could be cured through CESA and VASA. Our results offer novel insights into the treatment of urinary incontinence in women.

\section{MATERIALS AND METHODS}

The present study was conducted in 224 women with urinary incontinence who presented to the University Hospital of Cologne, Department of Obstetrics and Gynaecology, between January 2011 and December 2018. Only patients who never had undergone any surgery that could influence urinary incontinence were included in the study. Patients who had undergone colporrhaphy or a suburethral tape procedure were excluded from the study.

The diagnosis of urinary incontinence was established according to the recommendations of the International Continence Society by using established and validated urinary incontinence questionnaires. ${ }^{19,20,27}$

All the patients were additionally advised to perform a micturition protocol and maintain a specially designed bladder diary comprising questions on UUI symptoms for 12 days. Urodynamic measurements were performed only in the first year. However, treatment decisions were based on the clinical symptoms of the patients. A follow-up appointment was scheduled 2 weeks later for clinical examination, and an interview was conducted during this appointment.

The questions during the interviews comprised everyday situations to avoid misinterpretations or misunderstandings of the questionnaire. The following question was asked to differentiate patients with SUI and those with UUI:

"Do you lose urine when coughing or sneezing?"

When the answer to this question was affirmative, patients were categorized into SUI grade 1 (SUI 1). These patients were usually treated with a TOT. They were operated by CESA or VASA only when incontinence was associated with a descending uterus or vaginal stump.

To define UUI, the following question was asked to the patients:

"Imagine you are watching News on TV. Suddenly you feel the need to void urine. What do you do?"

The patients who gave the answer "When I get the feeling to void, I have to go immediately. Sometimes I reach the toilet in 
time, sometimes I already lose urine on my way to the toilet." and those who reported immediate urine loss with the feeling of urgency were classified as having UUI.

All patients with SUI and UUI were further asked the following questions to specify when they lost urine:

1) when still sitting on the chair?

2) when getting up from the chair?

3) on the way to the toilet?

4) other occasions?

When the answers to one or more of these questions were affirmative, patients were categorized as SUI grade 2 (SUI 2).

Thus, patients were categorized as SUI 1 and SUI 2. When they also had UUI symptoms, they were categorized as mixed urinary incontinence grade 1 (MUI 1) or MUI grade 2 (MUI 2). The corresponding answers were recorded both in a documentation sheet and electronically. The interviews were conducted during the initial presentation at the clinic and at 1 and 4 weeks postoperatively and the responses were documented accordingly.

Surgical treatment consisted of the CESA and VASA procedures. The corpus uteri was removed in patients undergoing CESA, and the CESA-structure (Dynamesh CESA, FEG, Aachen, Germany) was sutured to the cervical stump. The lateral USL parts "arms" were pulled through the peritoneal tunnel of the USL and fixed in front of the first sacral bone (S1). The "arms" were $8.8 \mathrm{~cm}$ in length for all patients. ${ }^{18}$

A similar procedure was followed in patients undergoing VASA. In patients who had hysterectomy the structure (Dynamesh VASA, FEG, Aachen, Germany) was sutured to the vaginal stump, and the "arms" were longer $(9.3 \mathrm{~cm}) .{ }^{17}$ The CESA and VASA operations are being performed through laparoscopy since 2016..$^{25,26}$

This study was approved by the Ethics Committee of the Medical Faculty of the University of Cologne, Germany (approval no: 201080).

\section{Statistical analysis}

The cure rate between groups was compared using a nonparametric test at a significance level of 5\%. Between-group analysis for categorical variables was performed using the $\chi^{2}$ or Fisher's exact tests. Pre- and post- scores were compared using the Wilcoxon test, and between-group comparisons of scores were performed using the Mann-Whitney $\mathrm{U}$ test. Binary logistic regression analyses were performed to study the influence of age on the treatment outcome. All analyses were performed using SPSS version 25 (SPSS, Chicago, III., USA) at a significance level of $5 \%$.

\section{RESULTS}

The study was conducted in 234 patients fulfilling the study criteria. Follow-up data of 10 patients were not available; and thus, the final analysis was performed on a total of 224 patients. According to our classification, 63 patients exhibited SUI 1 and 161 patients exhibited SUI 2. Of the 244 patients, 183 patients also presented with clinical symptoms of UUI and were considered as MUI. They were categorized either as MUI $1(n=39)$ or MUI $2(n=144)$ (Figure 1). Of the total patients, 128 patients (57.1\%) became continent after CESA or VASA (Figure 2). Of the total number of patients, $78 \%$ with SUI 1 (coughing and sneezing) became continent after CESA, and 63.6\% became continent after VASA (Figure 2). This difference was statistically significant $(p<0.05)$.

Of the 161 patients with SUI 2, the continence rate (CR) in patients was $56.2 \%$ after CESA and $45.7 \%$ after VASA (SUI 2) (Figure 2). However, this difference was not statistically significant ( $p>0.05)$. Of the 224 patients, 183 patients (81.6\%) with SUI were also suffering from urgency symptoms. These patients were classified as MUI 1 or MUI 2 (Figure 3). Of the 183 patients with MUI, 67 patients (36.6\%) became continent after CESA or VASA (Figure 3). Eighteen patients of 39 patients (46.1\%) with MUI 1 became continent and 49 patients of 144 patients with MUI 2 (34\%). This difference was not statistically significant $(p>0.05)$. The CR in patients with MUI 1 was $48 \%$ after CESA and $42 \%$ after VASA (Figure 3), whereas that in patients with MUI 2 was 39\% after CESA and 29\% after VASA. However, the difference was not statistically significant ( $p>0.05)$.

The CRs after CESA or VASA in patients with SUI were significantly higher than those in patients with MUI. This finding was observed in all subgroups of SUI as in MUI. The CR after CESA or

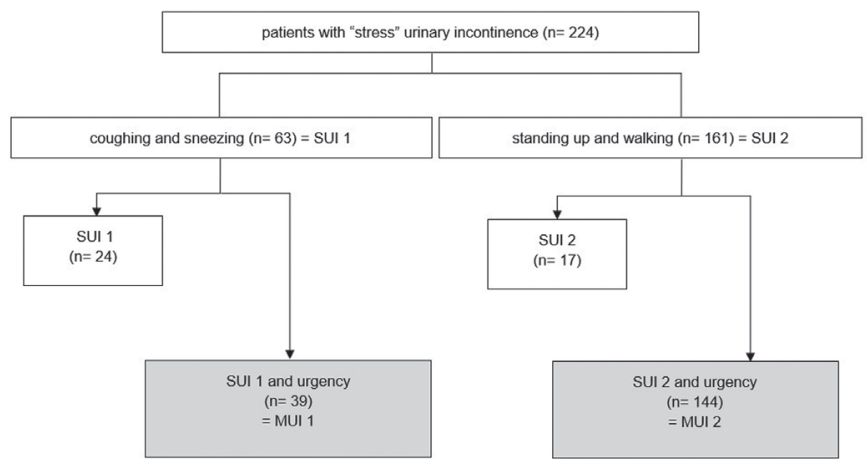

Figure 1. Classification of patients according to their urinary incontinence symptoms

Two hundred and twenty-four patients suffering from stress urinary incontinence (SUI) separated in patients who lose urine during different activities (SUI 1, coughing and sneezing; SUI 2, standing up and walking). Patients with additional urgency urinary incontinence (UUI) were referred to as mixed urinary incontinence (MUI) 


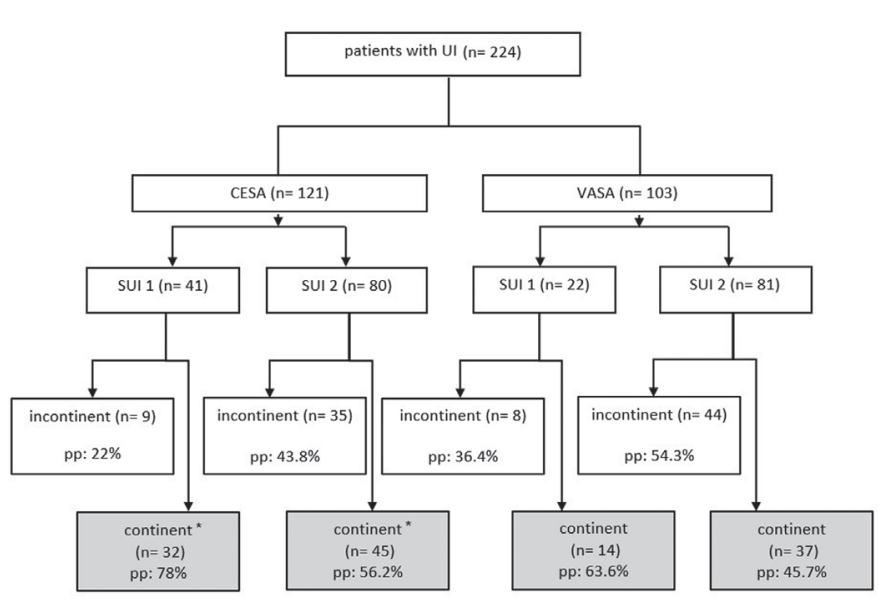

Figure 2. Effect of Cervicosacropexy (CESA) or Vaginosacropexy (VASA) on patients with stress urinary incontinence (SUI)

Patients suffering from SUI were separated in patients who lose urine with coughing and sneezing (SUI 1) or with standing up and walking (SUI 2). Ten patients with missing data (4 with CESA and 6 with VASA) were excluded.

pp: Per protocol, $n$ : Number

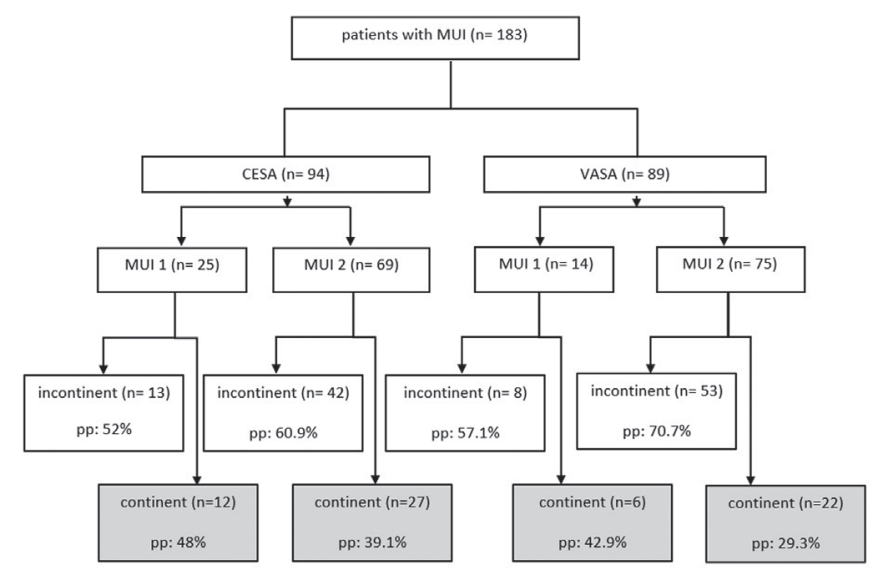

Figure 3. Effects of Cervicosacropexy (CESA) or Vaginosacropexy (VASA) on patients with mixed urinary incontinence (MUI)

Patients suffering from SUI with additional urgency urinary incontinence (UUI) were referred to as mixed urinary incontinence (MUI) and separated in patients who lose urine with coughing and sneezing (MUI 1) or with standing up and walking (MUI 2). Ten patients with missing data (4 with CESA and 6 with VASA) were excluded.

pp: per protocol, $n$ : Number

VASA in patients aged $<60$ years was $66 \%$, whereas the CR after CESA or VASA in patients aged $>60$ years was $48.7 \%$ (Figure 4 ), and the difference was statistically significant $(p<0.05)$. The $C R$ diminished with increase in age, and the least CR was observed in patients aged more than 70 years (Figure 5 ).

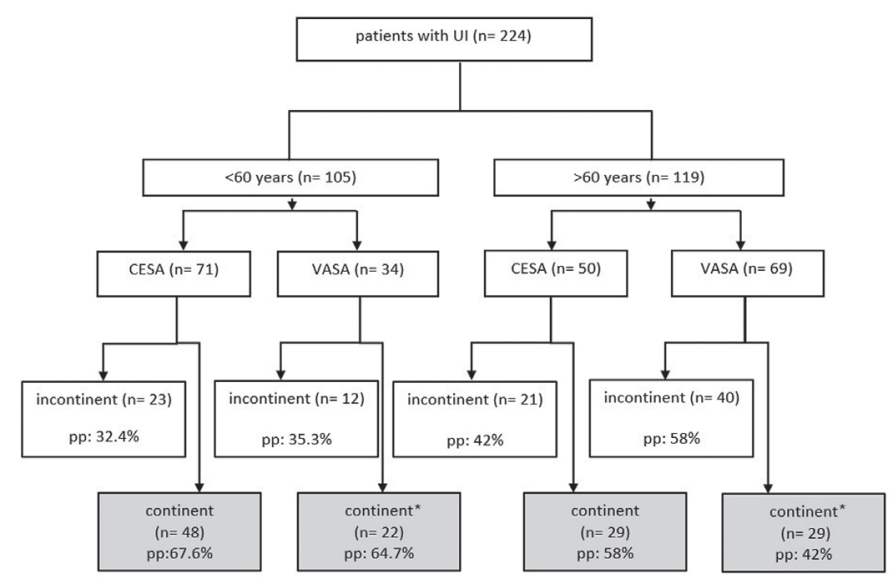

Figure 4. Continence rates (CR) of patients younger or older than 60 years of age at surgery

CESA: Cervicosacropexy; VASA: Vaginosacropexy; pp: Per protocol; $n$ : Number

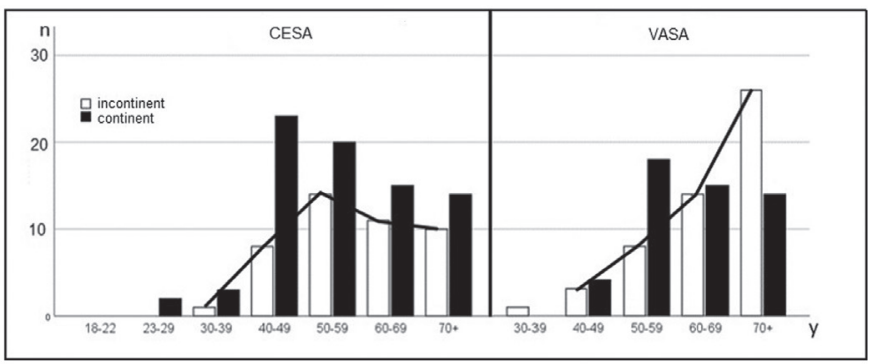

5. Number of urinary continent / incontinent patients after Cervicosacropexy (CESA) and Vaginosacropexy (VASA) according to different age groups at surgery

CESA: Cervicosacropexy; VASA: Vaginosacropexy

\section{DISCUSSION}

The present retrospective analysis of the effects of the apical fixation of the vagina on urinary incontinence led to the reconsideration of the current hypotheses on urinary incontinence in women. The study indicates that CESA and VASA can cure SUI, in addition to their effects on urgency. The elevation and tensioning of the upper vagina after CESA or VASA led to continence in patients having both SUI and MUI. ${ }^{7}$ Our previous studies have demonstrated that CESA or VASA can cure UUI. ${ }^{19,22-25}$ This finding was explained by the theory that the "urgency" transmitting receptors at the trigone of the bladder base adjacent to the UVJ are stabilized by the elevation or tensioning of the hammock.

However, CESA and VASA were also found to cure SUI, especially SUI during coughing or sneezing which is commonly believed as the indication for a TOT or tension-free vaginal tape. ${ }^{25,26}$

Effects on other "stress" (activity related) incontinence as incontinence when rising from a chair and walking to the toilet were less impressive but also found in about one third of 
patients. So far, we have no explanation for that effect of the apical fixation.

The strength of this study lies in the homogeneity of the clinical diagnosis and treatment in all the patients. We excluded patients with history of surgery that could have an influence on urinary continence. A hysterectomy could influence continence because the cervical support of the bladder is removed in this procedure. Therefore, data were separated between patients with a cervix (CESA) and those who had been hysterectomized (VASA).

All the patients underwent apical fixation through CESA or VASA. In CESA, the polyvinylidene difluoride tapes were sutured to the cervical stump (after supracervical hysterectomy) and fixed in front of the $S 1$ and were $8.8 \mathrm{~cm}$ long. In VASA, the tapes were sutured to the vaginal stump and were $9.3 \mathrm{~cm}$ long. The same lengths of the tapes could be used in all patients because the dimensions of the bony small pelvis are nearly identical in all women. ${ }^{22}$

The continence status was recorded preoperatively and at least two times postoperatively (at discharge and 4 weeks after discharge). The definition of continence was based on the experience and symptoms of the patients after CESA or VASA. All the patients were able to compare the symptoms before and after surgery. According to the standardized interview, the criteria for continence were always the same and reproducible by other investigators. Patients were considered continent, if they admitted that they "do not lose urine anymore! We can watch the News until the end and then go to the toilet without any loss of urine."

The CRs after CESA and VASA were different. More patients became continent after CESA than after VASA. However, the statistical analysis revealed that the difference was significant only in patients with SUI 1.

Significant differences in cure rates were observed in patients with urinary incontinence during different activities. Both CESA and VASA were able to cure more patients with SUI 1 than those with SUI 2, and the difference was statistically significant. In patients with severe "urgency" symptoms, outcomes in patients with MUI 2 were significantly worse compared with those having SUI 2 symptoms alone. Studies have demonstrated that the addition of a TOT can cure approximately $50 \%$ of patients who remain incontinent after CESA or VASA. It is not explained; however, why the other patients remain incontinent.

Our data indicated an inverse relation between age and the cure rate in both the patients with SUI and those with MUI. The cure rate in patients aged less than 60 years with SUI symptoms was significantly higher than that in patients aged more than 60 years. Similar findings were observed in patients with MUI.
We hypothesize that urinary incontinence is a pathophysiological continuum. Pressure plays a vital role in incontinence. When the bladder is empty, no "urgency" exists; the "urgency" comes with the filling of the bladder which causes an increase in pressure on the bladder wall and probably on the UVJ and the "urgency" receptors. Incontinence, however, occurs when the patient stands up from the sitting position. In that moment, the counterpressure of the perineum on the UVJ diminishes, the UVJ opens, and the patient loses urine. A study by Jäger et al. reported that the association between "urgency" and activity-related incontinence is observed in 96\% of all patients with UUI. ${ }^{19}$ The finding could explain the role of medical treatment in decreasing the urgency symptoms; however, not the incontinence episodes. According to the International Urogynaecological Association (IUGA), UUI is defined as the complaint of involuntary loss of urine associated with urgency, and SUI is defined as the complaint of the involuntary loss of urine during effort or physical exertion. ${ }^{30}$ Therefore, the IUGA recommended that the term "activity-related incontinence" should be preferred to "SUI" to differentiate SUI from psychological stress.

Thus, according to the recommended definitions of incontinence by the IUGA, all patients with or without MUI were suffering from "activity-related incontinence." The preferred treatment for this type of incontinence is surgical and not medical.

This may explain why a surgical treatment such as CESA and VASA led to continence and disappearance of the "urgency." Thus, the feeling of "urgency" is based on a change in anatomy, which can be repaired by the respective surgical treatment.

\section{CONCLUSIONS}

The greatest advantage of this study is its reproducibility. CESA and VASA are the standardized operations that can be performed similarly by every surgeon. Furthermore, the symptoms of urinary incontinence were based on the subjective complaints of the patients. "Urgency" is basically a symptom and can also be evaluated according to our interview method. Therefore, the results presented in this study can be proven or disproven by every surgeon. From the scientific point of view, this is a crucial message.

\section{Contributions}

Study design: W.J. and P.M., Data collection: W.J. and S.L., Analysis of Data: A.B., Manuscript writing: W.J.

\section{Ethics}

Ethics Committee Approval: This study was approved by the Ethics Committee of the Medical Faculty of the University of Cologne, Germany (approval no: 19-1229). 
Informed Consent: It was obtained.

Peer-review: Externally peer-reviewed.

Conflict of Interest: Wolfram Jäger and Sebastian Ludwig receive honoraria from the FEG Textiltechnik mbH, Aachen, Germany. No conflict of the interest was declared by other authors.

Financial Disclosure: Wolfram Jäger and Sebastian Ludwig receive honoraria from the FEG Textiltechnik mbH, Aachen, Germany. The other authors state that they did not receive any financial support for the study and that they have no personal, political or religious interests which interfere with the study.

\section{REFERENCES}

1. Petros PE, Ulmsten UI. An integral theory of female urinary incontinence. Experimental and clinical considerations. Acta Obstet Gynecol Scand Suppl 1990; 153: 7-31.

2. Petros PE, Ulmsten UI. An integral theory and its method for the diagnosis and management of female urinary incontinence. Scand J Urol Nephrol Suppl 1993; 153: 1-93.

3. Petros PP, Ulmsten U. Urge incontinence history is an accurate predictor of urge incontinence. Acta Obstet Gynecol Scand 1992; 71: 537-539.

4. Ulmsten U. Some reflections and hypotheses on the pathophysiology of female urinary incontinence. Acta Obstet Gynecol Scand Suppl 1997; 166: 3-8.

5. Rezapour M, Falconer C, Ulmsten U. Tension-Free vaginal tape (TVT) in stress incontinent women with intrinsic sphincter deficiency (ISD)--a long-term follow-up. Int Urogynecol J Pelvic Floor Dysfunct 2001; 12: 12-14.

6. Petros P. Creating a gold standard surgical device: scientific discoveries leading to TVT and beyond: UIf Ulmsten Memorial Lecture 2014. Int Urogynecol J 2015; 26: 471-476.

7. de Boer TA, Gietelink DA, Hendriks JC, Vierhout ME. Factors influencing success of pelvic organ prolapse repair using porcine dermal implant Pelvicol. Eur J Obstet Gynecol Reprod Biol 2010; 149: $112-116$.

8. de Boer TA, Slieker-ten Hove MC, Burger CW, Vierhout ME. The prevalence and risk factors of overactive bladder symptoms and its relation to pelvic organ prolapse symptoms in a general female population. Int Urogynecol J 2011; 22: 569-575.

9. Chen L, Ramanah R, Hsu Y, Ashton-Miller JA, Delancey JO. Cardinal and deep uterosacral ligament lines of action: MRI based 3D technique development and preliminary findings in normal women. Int Urogynecol J 2013; 24: 37-45.

10. Alhasso AA, McKinlay J, Patrick K, Stewart L. Anticholinergic drugs versus non-drug active therapies for overactive bladder syndrome in adults. Cochrane Database Syst Rev 2006; (4): CD003193.

11. Abrams P, Andersson KE, Birder L, et al. Fourth International Consultation on Incontinence. Fourth International Consultation on Incontinence Recommendations of the International Scientific Committee: Evaluation and treatment of urinary incontinence, pelvic organ prolapse, and fecal incontinence. Neurourol Urodyn 2010; 29: 213-240.

12. Dubeau CE, Kraus SR, Griebling TL, et al. Effect of fesoterodine in vulnerable elderly subjects with urgency incontinence: a doubleblind, placebo controlled trial. J Urol 2014; 191: 395-404.

13. Siddiqui NY, Geller EJ, Visco AG. Symptomatic and anatomic 1-year outcomes after robotic and abdominal sacrocolpopexy. Am J Obstet Gynecol 2012; 206: 435.e1-5.

14. Thangarajah F, Hartmann-Wobbe J, Ratiu D, et al. The Onset of Urinary Incontinence in Different Subgroups and its Relation to Menopausal Status: A Hospital-based Study. In Vivo 2020; 34: 923928.

15. Bourrat M, Armand C, Seffert P, Tostain J. Complications et résultats fonctionnels à moyen terme du TVT dans l'incontinence urinaire d'effort [Complications and medium-term functional results of TVT in stress urinary incontinence]. Prog Urol 2003; 13: 1358-1364.

16. Koops SE, Bisseling TM, van Brummen HJ, Heintz AP, Vervest HA. What determines a successful tension-free vaginal tape? A prospective multicenter cohort study: results from The Netherlands TVT database. Am J Obstet Gynecol 2006; 194: 65-74.

17. Jaeger W. Vagino-Sacropexy (VASA) and Vagino-Recto-Sacropexy (VARESA). In: Alkatout I and Mettler L, eds. Hysterectomy - A Comprehensive Surgical Approach. Cham, Switzerland: Springer International Publishing. 2016. p. 1137-1157.

18. Jaeger W. Cervico-Sacropexy (CESA) and Cervico-Recto-Sacropexy (CERESA). In: Alkatout I and Mettler L, eds. Hysterectomy - A Comprehensive Surgical Approach. Cham, Switzerland: Springer International Publishing. 2016. p. 1117-1136.

19. Jaeger W, Ludwig S, Neumann E, Mallmann P. Evidence of Common Pathophysiology Between Stress and Urgency Urinary Incontinence in Women. In Vivo 2020; 34: 2927-2932.

20. Jaeger W, Ludwig S, Stumm M, Mallmann P. Standardized bilateral mesh supported uterosacral ligament replacement - cervicosacropexy (CESA) and vagino-sacropexy (VASA) operations for female genital prolapse. Pelviperineology 2016; 35: 1-5.

21. Jäger W, Mirenska 0 , Brügge $S$. Surgical treatment of mixed and urge urinary incontinence in women. Gynecol Obstet Invest 2012; 74: 157-164.

22. Rizk DE, Czechowski J, Ekelund L. Dynamic assessment of pelvic floor and bony pelvis morphologic condition with the use of magnetic resonance imaging in a multiethnic, nulliparous, and healthy female population. Am J Obstet Gynecol 2004; 191: 83-89.

23. Cassis C, Mukhopadhyay S, Morris E. Standardizing abdominal sacrocolpopexy for the treatment of apical prolapse: One year on. Int J Gynaecol Obstet 2019; 147: 49-53.

24. Rajshekhar S, Mukhopadhyay S, Morris E. Early safety and efficacy outcomes of a novel technique of sacrocolpopexy for the treatment of apical prolapse. Int J Gynaecol Obstet 2016; 135: 182-186.

25. Ludwig S, Becker I, Mallmann P, Jäger W. Comparison of Solifenacin and Bilateral Apical Fixation in the Treatment of Mixed and Urgency Urinary Incontinence in Women: URGE 1 Study, A Randomized Clinical Trial. In Vivo 2019; 33: 1949-1957.

26. Rexhepi S, Rexhepi E, Stumm M, Mallmann P, Ludwig S. Laparoscopic Bilateral Cervicosacropexy and Vaginosacropexy: New Surgical 
Treatment Option in Women with Pelvic Organ Prolapse and Urinary Incontinence. J Endourol 2018; 32: 1058-1064.

27. Abrams P, Cardozo L, Wein A. 3rd International Consultation on Incontinence--Research Society 2011. Neurourol Urodyn 2012; 31: 291-292.
28. Haylen BT, de Ridder D, Freeman RM, et al. An International Urogynecological Association (IUGA)/International Continence Society (ICS) joint report on the terminology for female pelvic floor dysfunction. Int Urogynecol J 2010; 21: 5-26. 